\title{
Urban River Reserve Potentials as Passive Cooling Strategies
}

\author{
Siti Rahmah Omar ${ }^{1 *}$, Johan Sohaili ${ }^{1}$, Nur Hidayah Abd Rahman ${ }^{2}$, Norhazren Izatie Mohd ${ }^{3}$ \\ ${ }^{1}$ Faculty of Engineering, Universiti Teknologi Malaysia, 81310 Skudai, Johor, Malaysia \\ ${ }^{2}$ Kulliyyah of Languages and Management, International Islamic University Malaysia, 84600, Pagoh, \\ Johor, Malaysia \\ ${ }^{3}$ Faculty of Built Environment and Surveying, Universiti Teknologi Malaysia, 81310 Skudai, Johor, \\ Malaysia
}

\begin{abstract}
In Malaysia, urban riparian refers to river reserve that reflects green space. As surviving greenery space amidst concrete jungle, urban riparian is essential for safe-keeping the river health, the surrounding environment, and the well-being of urbanites. The existing developments found by the Malaysian riversides are already in place prior to river reserves establishment, thus constricting expansion and synchronisation due to its guidelines applied in isolation within several related agencies. Prior studies have highlighted the potential of riparian vegetation as a viable method that lowers the temperature within urban space. Hence, this paper probes into the potential of river reserve as a passive cooling strategy by determining the vegetation technical requirements from the light of urban river physical planning and design guidelines in Malaysia. Thus, this study outlines the possibility of integration by comparing vegetation requirements for river buffer with vegetation function and ability in altering radiation, as well as heat transfer, through physical attributes. By focusing on vegetation physical structure, the riparian area can attain multiple functions and benefits. Thus, this finding serves as a basis for integration of passive cooling strategies with vegetation requirements for urban river reserve, and for further studies that investigate other essential physical attributes.
\end{abstract}

\section{Introduction}

It is undeniable that the increasing population of humanity has an impact on the urban environment. One effective way to minimise the impact is by preserving and conserving urban vegetation. Urban vegetation has a significant role that has been proven to be a major strategy in mitigating river pollution $[1,2,3]$, apart from enhancing the conditions of urban thermal $[4,5]$. Within the Malaysian context, urban riparian is also termed as river reserve that has been reckoned as green space. In its function as surviving greenery in an urban area [6], urban riparian has essential roles in the river health, the surrounding environment, and

\footnotetext{
* Corresponding author: $\underline{\text { ct.rahmah83@gmail.com }}$
} 
the well-being among urbanites. The existing developments found along the Malaysian riversides have been in place prior to establishment of river reserves [7], thus leading to expansion constraint. Furthermore, the rising concern and issues related to the river conditions in Malaysia have been long discussed [8, 9, 10, 11], and improvement of the overall urban rivers is yet to be realised. Therefore, by adopting the best possible solution to the constricted urban riparian space in light of its vegetation structure, two necessities could be tackled at once, apart from maximising the benefits that may be reaped from this limited strip of land.

As such, the government has to revise the existing regulations that control river pollution and riverfront developments in Malaysia, so as to aid the government facilitate, control, and enforce the best practice for riverfront developments in Malaysia towards achieving successful river management and riverfront outcomes [12, 13, 14]. Additionally, past studies have emphasised the potential of riparian vegetation as an effective strategy to decrease the rising temperatures in urban areas [15, 16, 17]. With that, this paper determined the potential of urban river reserve vegetation as a passive cooling strategy [18, 19] by comparing the vegetation technical requirement for river buffer with its physical structure, as well as its ability in altering heat transfer mechanisms. This approach should be able to facilitate riparian area in attaining its multiple functions and benefits, particularly for its vegetation physical structure.

\section{Methodology}

The method of content analysis had been employed in this study in order to identify the types and the specification of vegetation in terms of function for riparian buffer and thermal reduction. Content analysis refers to a research technique for an objective, systematic, and quantitative description of the manifested content of communication that enables one to study the interpretation of messages from various media, such as books, articles, magazines, reports, speeches, web pages, and other relevant materials [20]. The method of content analysis can be divided into two types; deductive and inductive. The deductive approach focuses on objectivism and existing knowledge that pre-determine the coding extracted from findings in the literature review. On the other hand, the inductive approach places more weight on the subjective aspect, which is qualitative in nature. The content is extracted from open coding to arrive at categories and abstraction [21, 22]. The outcomes only pursue the basic context, thus resulting in similar findings for each document or message [23]. As for this particular study, the analysed content components had been comprised of trees, shrubs, and grass [24]. All vegetation technical requirements and statements, which are relevant to urban river physical planning and design guidelines in Malaysia [25], were identified and listed. The guidelines were retrieved from the Malaysian Department of Irrigation and Drainage (DID), the Malaysian Ministry of Natural Resources and Environment (NRE), National Landscape Department (NLD), and Town and Country Planning Department (TCPD) for Peninsular Malaysia.

This paper, hence, lists the vegetation types and specification that can minimise outdoor thermal, which refers to a passive cooling strategy [18, 19]. For both guidelines and published articles, all general statements that did not specify vegetation types or specification (e.g. area, height, density, and form), as well as all biodiversity requirements, had been excluded from this study. The relevant research materials were collected from the Web of Science and SCOPUS databases. As a result, the materials retrieved consisted of five riparian-related guidelines and twelve articles from 1991 to 2017. Although the sampling seems small, Uribe and Manzur [26] asserted that a sampling size that ranges between 6 and 12 is sufficient for a content analysis study. Finally, all vegetation functions and benefits were simplified and tabulated to identify the similarities by comparing the 
vegetation requirement for river buffer with its ability to alter radiation and heat transfer through its physical attributes. This should be able to facilitate the riparian area towards achieving its multiple functions and benefits, especially from the physical structure of the vegetation.

\section{Results and discussion}

All the findings and statements associated to trees, shrubs, and grass are tabulated in Table 1. The riparian-related guidelines [27-31] seemed to have more focus on tree-covered areas, along with their width. As for shrubs and grass, the specification emphasised on density, area width, and covered area. Except for grass, the overall height specification for trees and shrubs were omitted for riparian buffer zone requirements. This could probably due to the focus and the objective of the urban riparian buffer, which serves as a mechanism to control non-point source surface run-off and bank stabilisation. Furthermore, from these retrieved guidelines, nine significant functions of urban riparian had been determined, which are: 1) stabilises banks, 2) prevents erosion, 3) traps sediments, 4) filters pollutants and nutrients, 5) provides flood storage and conveyance, 6) slows surface run-off, 7) shades watercourse, 8) recharges groundwater, and lastly, 9) buffers zone (as tabulated in Tables 1 and 2).

Based on the guidelines retrieved, it is indicative that a good urban riparian should consist of the listed functions for the benefit of the river, as well as its water quality. These outcomes also highlight the effects and the advantages of microclimate, which appear to be lacking in the Malaysian urban riparian. In addition, articles that asserted the impact of vegetation types and specification upon thermal reductions [32-43] pointed out the higher importance of tree specification (see Table 2). Tree specifications include tree height, trunk height, canopy width, canopy height, canopy density, and covered area. As for shrubs and grass, their specification is only limited to covered area.

Table 1. Vegetation specifications and their specific functions.

\begin{tabular}{|l|l|l|l|l|}
\hline Source & $\begin{array}{l}\text { Vegetation } \\
\text { Type }\end{array}$ & Specification & Function & \multicolumn{1}{|c|}{ Descriptions / Statements } \\
\hline$[27]$ & Tree & Tree Cover & $\begin{array}{l}\text { Offers shade } \\
\text { for } \\
\text { watercourse } \\
\text { /protection }\end{array}$ & $\begin{array}{l}\text { Cutting trees decrease protection. } \\
\text { Various types of river management } \\
\text { works can provide or protect shady } \\
\text { areas, such as planting small trees } \\
\text { along riverbanks. }\end{array}$ \\
\hline & Grass & Area Width & $\begin{array}{l}\text { Buffer Zone / } \\
\text { Barrier }\end{array}$ & $\begin{array}{l}\text { Under normal circumstances, a space } \\
\text { of 5 m is sufficient (for the river } \\
\text { within a city). This space depends on } \\
\text { the state of the river involved and the } \\
\text { needs of DID. This space should only } \\
\text { be open and must be planted with } \\
\text { grass as a buffer zone. }\end{array}$ \\
\hline Grass & $\begin{array}{l}\text { Covered } \\
\text { Area }\end{array}$ & $\begin{array}{l}\text { Stabilises } \\
\text { Riverbank / } \\
\text { Prevents } \\
\text { erosion }\end{array}$ & $\begin{array}{l}\text { If the plants are well-maintained, the } \\
\text { creeping roots will bind and reinforce } \\
\text { the riverbank. The grass layers } \\
\text { planted on the riverbanks will be able } \\
\text { to withstand erosion from the stream. }\end{array}$ \\
\hline$[28]$ & Tree & $\begin{array}{l}\text { Covered } \\
\text { Area }\end{array}$ & $\begin{array}{l}\text { Surface run- } \\
\text { off / Prevents } \\
\text { erosion }\end{array}$ & $\begin{array}{l}\text { Tree (Forest) covered area affects } \\
\text { run-off estimation. } \\
\text { Tree covers provide long-term } \\
\text { stabilisation of soil. }\end{array}$ \\
\hline & Tree & Area Width & Stabilises & 10-20m width reduces bank erosion. \\
\hline
\end{tabular}




\begin{tabular}{|c|c|c|c|c|}
\hline Source & $\begin{array}{c}\text { Vegetation } \\
\text { Type }\end{array}$ & Specification & Function & Descriptions / Statements \\
\hline & & & $\begin{array}{l}\text { Riverbank / } \\
\text { Flood storage } \\
\text { and } \\
\text { conveyance }\end{array}$ & $\begin{array}{l}20-150 \mathrm{~m} \text { width increases water flow } \\
\text { time by interception, resulting in } \\
\text { reduced flood peaks. }\end{array}$ \\
\hline & Shrub & Area Width & $\begin{array}{l}\text { Stabilises } \\
\text { Riverbank / } \\
\text { Flood storage } \\
\text { and } \\
\text { conveyance }\end{array}$ & $\begin{array}{l}10-20 \mathrm{~m} \text { width reduces bank erosion. } \\
20-150 \mathrm{~m} \text { width increases water flow } \\
\text { time by interception, resulting in } \\
\text { reduced flood peaks. }\end{array}$ \\
\hline & & Density & $\begin{array}{l}\text { Surface run- } \\
\text { off / Traps } \\
\text { Sediments / } \\
\text { Filters } \\
\text { pollutants / } \\
\text { Recharges } \\
\text { groundwater }\end{array}$ & $\begin{array}{l}\text { Dense herbaceous buffers with } \\
\text { recommended width intercept run-off, } \\
\text { trap sediments, remove pollutants, } \\
\text { and promote ground water recharge. }\end{array}$ \\
\hline & & $\begin{array}{l}\text { Covered } \\
\text { Area }\end{array}$ & $\begin{array}{l}\text { Surface run- } \\
\text { off / Prevents } \\
\text { erosion }\end{array}$ & $\begin{array}{l}\text { Shrub-covered area affects run-off } \\
\text { estimation. } \\
\text { Shrubs covers provide long-term } \\
\text { stabilisation of soil. }\end{array}$ \\
\hline & Grass & Density & 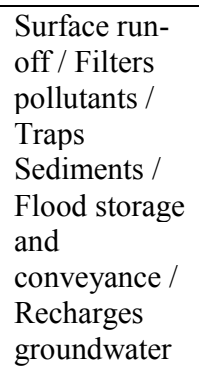 & $\begin{array}{l}\text { Densely-grassed surface affects travel } \\
\text { time of run-off flows, (Horton's } \\
\text { Roughness). } \\
\text { Dense grass filters mechanism. } \\
\text { Dense grass buffers (recommended } \\
\text { width) intercept run-off, trap } \\
\text { sediments, remove pollutants, reduce } \\
\text { flow, and promote groundwater } \\
\text { recharge. }\end{array}$ \\
\hline & & $\begin{array}{l}\text { Overall } \\
\text { Height }\end{array}$ & $\begin{array}{l}\text { Surface run- } \\
\text { off / Filters } \\
\text { pollutants / } \\
\text { Traps } \\
\text { Sediments }\end{array}$ & $\begin{array}{l}\text { Grass cover height affects travel time } \\
\text { of run-off flows (Manning } \\
\text { Roughness). Short Grass Cover is }< \\
150 \mathrm{~mm} \text { and Tall Grass Cover } \geq 150 \\
\mathrm{~mm} \text {. } \\
\text { Grass height as a factor for } \\
\text { sedimentation and water quality } \\
\text { treatment. }\end{array}$ \\
\hline & & $\begin{array}{l}\text { Covered } \\
\text { Area }\end{array}$ & $\begin{array}{l}\text { Surface run- } \\
\text { off / Prevents } \\
\text { erosion / } \\
\text { Traps } \\
\text { sediments }\end{array}$ & $\begin{array}{l}\text { Grass-covered area affects run-off } \\
\text { estimation. } \\
\text { Grass coverage as a factor in erosion } \\
\text { control treatment and in reducing } \\
\text { sediment loading. }\end{array}$ \\
\hline & & Area Width & $\begin{array}{l}\text { Stabilises } \\
\text { bank / Flood } \\
\text { storage and } \\
\text { conveyance }\end{array}$ & $\begin{array}{l}10-20 \mathrm{~m} \text { width reduces bank erosion. } \\
20-150 \mathrm{~m} \text { width increases water flow } \\
\text { time by interception, resulting in } \\
\text { reduced flood peaks. }\end{array}$ \\
\hline [29] & Tree & $\begin{array}{l}\text { Covered } \\
\text { Area }\end{array}$ & $\begin{array}{l}\text { Shade for } \\
\text { watercourse / } \\
\text { Stabilises } \\
\text { bank }\end{array}$ & $\begin{array}{l}\text { Trees provide shade that reduces } \\
\text { water temperature. } \\
\text { Trees are highly effective in } \\
\text { preventing bank failures. }\end{array}$ \\
\hline & & Area Width & $\begin{array}{l}\text { Stabilises } \\
\text { bank }\end{array}$ & $\begin{array}{l}\text { 10m width (minimum) for bank } \\
\text { stabilisation. }\end{array}$ \\
\hline & Shrub & Covered & Shade for & Shrubs provide shade to reduce water \\
\hline
\end{tabular}




\begin{tabular}{|c|c|c|c|c|}
\hline Source & $\begin{array}{l}\text { Vegetation } \\
\text { Type }\end{array}$ & Specification & Function & Descriptions / Statements \\
\hline & & Area & $\begin{array}{l}\text { watercourse / } \\
\text { Stabilises } \\
\text { bank }\end{array}$ & $\begin{array}{l}\text { temperature. } \\
\text { Shrubs are highly effective in } \\
\text { stabilising riverbank. }\end{array}$ \\
\hline & & Area Width & $\begin{array}{l}\text { Stabilises } \\
\text { bank }\end{array}$ & $\begin{array}{l}10 \mathrm{~m} \text { width (minimum) for bank } \\
\text { stabilisation. }\end{array}$ \\
\hline & Grass & Area Width & $\begin{array}{l}\text { Trap } \\
\text { sediments / } \\
\text { Filter } \\
\text { nutrients / } \\
\text { Surface run- } \\
\text { off }\end{array}$ & $\begin{array}{l}\text { Highly effective to Trap sediment, } \\
\text { Filter sediment-bound nutrients, } \\
\text { pesticides, and microbes. } \\
\text { Converts surface run-off to uniform, } \\
\text { shallow, and sheet flow. }\end{array}$ \\
\hline \multirow[t]{4}{*}[30]{} & Tree & Covered area & Shading & Trees that can provide shade. \\
\hline & Shrub & Covered area & $\begin{array}{l}\text { Buffer Zone / } \\
\text { Barrier }\end{array}$ & $\begin{array}{l}\text { Shrubs need to be planted closely to } \\
\text { produce fencing effect. }\end{array}$ \\
\hline & & Density & $\begin{array}{l}\text { Buffer Zone / } \\
\text { Barrier }\end{array}$ & $\begin{array}{l}\text { Shrub (with dense and lush leaf } \\
\text { texture) as buffer zone. }\end{array}$ \\
\hline & Grass & Area Width & $\begin{array}{l}\text { Trap } \\
\text { sediment / } \\
\text { Filter } \\
\text { nutrients, } \\
\text { pesticides. } \\
\text { and microbes }\end{array}$ & $\begin{array}{l}20 \mathrm{~m}-30 \mathrm{~m} \text { width to control the effects } \\
\text { of silt, fertilisation, and insecticides }\end{array}$ \\
\hline$[31]$ & Tree & Covered area & $\begin{array}{l}\text { Stabilises } \\
\text { bank }\end{array}$ & $\begin{array}{l}\text { The trees that suit the river habitat are } \\
\text { able to strengthen the riverbank. }\end{array}$ \\
\hline \multirow[t]{3}{*}{ [32] } & Tree & Height & $\begin{array}{l}\text { Reduces } \\
\text { solar } \\
\text { radiation and } \\
\text { Mean } \\
\text { Radiant } \\
\text { Temperature }\end{array}$ & $\begin{array}{l}\text { Tall trees in deeper urban canyon and } \\
\text { vice versa for thermal comfort } \\
\text { improvement in urban area. }\end{array}$ \\
\hline & & Trunk Height & $\begin{array}{l}\text { Improves } \\
\text { Ventilation }\end{array}$ & $\begin{array}{l}\text { Higher trunk height allows improved } \\
\text { ventilation and traffic pollutant } \\
\text { dispersion. }\end{array}$ \\
\hline & & $\begin{array}{l}\text { Canopy } \\
\text { density }\end{array}$ & $\begin{array}{l}\text { Reduces } \\
\text { solar } \\
\text { radiation and } \\
\text { Mean } \\
\text { Radiant } \\
\text { Temperature }\end{array}$ & $\begin{array}{l}\text { Less canopy density in deeper urban } \\
\text { canyon and vice versa for thermal } \\
\text { comfort improvement in urban area. }\end{array}$ \\
\hline \multirow[t]{2}{*}{ [33] } & Tree & $\begin{array}{l}\text { Canopy } \\
\text { Height }\end{array}$ & $\begin{array}{l}\text { Influences } \\
\text { wind speed }\end{array}$ & $\begin{array}{l}\text { For a measurement point at which the } \\
\text { wind speeds were measured at two } \\
\text { heights, the ventilation was even } \\
\text { worse at the height of } 4.5 \mathrm{~m} \text { due to } \\
\text { tree crown obstruction, as compared } \\
\text { to that at the height of } 1.5 \mathrm{~m} \text {. }\end{array}$ \\
\hline & & $\begin{array}{l}\text { Canopy } \\
\text { density }\end{array}$ & $\begin{array}{l}\text { Influences } \\
\text { wind speed }\end{array}$ & $\begin{array}{l}\text { Excessively dense planting without } \\
\text { considering wind corridor in the park } \\
\text { that obstructs air flow and rises } \\
\text { temperature at downstream areas. }\end{array}$ \\
\hline [34] & Tree & $\begin{array}{l}\text { Covered } \\
\text { Area }\end{array}$ & $\begin{array}{l}\text { Reduces } \\
\text { Surface and }\end{array}$ & $\begin{array}{l}\text { Tree shade reduces surface and global } \\
\text { temperatures by up to } 19^{\circ} \mathrm{C} \text { and } 5-\end{array}$ \\
\hline
\end{tabular}




\begin{tabular}{|c|c|c|c|c|}
\hline Source & $\begin{array}{c}\text { Vegetation } \\
\text { Type }\end{array}$ & Specification & Function & Descriptions / Statements \\
\hline & & & $\begin{array}{l}\text { Global } \\
\text { Temperatures }\end{array}$ & $7 \circ \mathrm{C}$, respectively. \\
\hline & Grass & $\begin{array}{l}\text { Covered } \\
\text { Area }\end{array}$ & $\begin{array}{l}\text { Reduces } \\
\text { Surface } \\
\text { Temperature }\end{array}$ & $\begin{array}{l}\text { Grass reduces maximum surface } \\
\text { temperature by up to } 24^{\circ} \mathrm{C} \text {. }\end{array}$ \\
\hline \multirow[t]{2}{*}{ [35] } & Tree & $\begin{array}{l}\text { Covered } \\
\text { Area }\end{array}$ & $\begin{array}{l}\text { Reduces } \\
\text { Surface, } \\
\text { Global, and } \\
\text { Air } \\
\text { Temperatures }\end{array}$ & $\begin{array}{l}\text { Trees have the largest effect on } \\
\text { surface, global, and air temperatures } \\
\text { when compared to any other } \\
\text { parameter. }\end{array}$ \\
\hline & Grass & $\begin{array}{l}\text { Covered } \\
\text { Area }\end{array}$ & $\begin{array}{l}\text { Reduces } \\
\text { Surface, } \\
\text { Global, and } \\
\text { Air } \\
\text { Temperatures }\end{array}$ & $\begin{array}{l}\text { Exposed grass surface is cooler than } \\
\text { hard pavements and has lower global } \\
\text { temperature. Shaded grass surfaces } \\
\text { are very significantly cooler than the } \\
\text { respective exposed surfaces. } \\
\text { Grass surfaces in the shade of trees } \\
\text { have considerably lower surface, } \\
\text { global, and air temperatures. }\end{array}$ \\
\hline \multirow[t]{2}{*}{ [36] } & Tree & $\begin{array}{l}\text { Canopy } \\
\text { Density }\end{array}$ & $\begin{array}{l}\text { Reduces } \\
\text { Solar } \\
\text { Radiation, } \\
\text { Reflected } \\
\text { Radiation, } \\
\text { Air } \\
\text { Temperature, } \\
\text { and Surface } \\
\text { Temperature }\end{array}$ & $\begin{array}{l}\text { M. ferrea L. is more significant as a } \\
\text { thermal radiation filter than H. } \\
\text { crepitans L., due to the former's } \\
\text { denser foliage cover and branching } \\
\text { habit. This significant filtration } \\
\text { capability reduces terrestrial } \\
\text { radiation, cools the ground surfaces } \\
\text { by promoting more latent heat, and } \\
\text { decreases air temperature. }\end{array}$ \\
\hline & & $\begin{array}{l}\text { Canopy } \\
\text { Width }\end{array}$ & $\begin{array}{l}\text { Reduces } \\
\text { Solar } \\
\text { radiation }\end{array}$ & $\begin{array}{l}\text { Canopies with larger width create } \\
\text { more shadow and a wider shaded } \\
\text { area. }\end{array}$ \\
\hline \multirow[t]{5}{*}[37]{} & Tree & Trunk height & Ventilation & $\begin{array}{l}\text { A single isolated tree, especially with } \\
\text { a high trunk, may concentrate the } \\
\text { airflow below the canopy and } \\
\text { improve ventilation near the ground. }\end{array}$ \\
\hline & & $\begin{array}{l}\text { Canopy } \\
\text { Height }\end{array}$ & $\begin{array}{l}\text { Reduces } \\
\text { solar } \\
\text { radiation / } \\
\text { Wind } \\
\text { Movement }\end{array}$ & $\begin{array}{l}\text { Trees with high canopy near walls } \\
\text { and windows provide shade and } \\
\text { reduce solar heat gain with relatively } \\
\text { small blockage of the wind. }\end{array}$ \\
\hline & & $\begin{array}{l}\text { Canopy } \\
\text { Density }\end{array}$ & $\begin{array}{l}\text { Wind } \\
\text { Movement }\end{array}$ & $\begin{array}{l}\text { A dense canopy of trees along streets } \\
\text { can reduce the street level wind speed } \\
\text { significantly. }\end{array}$ \\
\hline & Shrub & $\begin{array}{l}\text { Covered } \\
\text { Area }\end{array}$ & $\begin{array}{l}\text { Wind } \\
\text { Movement }\end{array}$ & $\begin{array}{l}\text { Evergreen shrubs along trees prevent } \\
\text { wind penetration below the canopy. }\end{array}$ \\
\hline & Grass & $\begin{array}{l}\text { Covered } \\
\text { Area }\end{array}$ & $\begin{array}{l}\text { Wind } \\
\text { Movement }\end{array}$ & $\begin{array}{l}\text { Grassy areas pose the least friction } \\
\text { (resistance) to airflow and allow the } \\
\text { best possible ventilation conditions. }\end{array}$ \\
\hline \multirow[t]{2}{*}{ [38] } & Tree & $\begin{array}{l}\text { Canopy } \\
\text { Width }\end{array}$ & $\begin{array}{l}\text { Reduces } \\
\text { solar } \\
\text { radiation }\end{array}$ & $\begin{array}{l}\text { In a green area with trees, the cooling } \\
\text { effect is determined by the amount of } \\
\text { canopy shading. }\end{array}$ \\
\hline & & $\begin{array}{l}\text { Covered } \\
\text { Area }\end{array}$ & $\begin{array}{l}\text { Reduces } \\
\text { solar } \\
\text { radiation }\end{array}$ & $\begin{array}{l}\text { Complete shading coverage from the } \\
\text { canopy can have an average cooling } \\
\text { effect of } 3.23 \mathrm{~K} \text { at all sites. }\end{array}$ \\
\hline [39] & Tree & Covered & Reduces & Tree contributes to comfort by \\
\hline
\end{tabular}




\begin{tabular}{|c|c|c|c|c|}
\hline Source & $\begin{array}{c}\text { Vegetation } \\
\text { Type }\end{array}$ & Specification & Function & Descriptions / Statements \\
\hline & & Area & $\begin{array}{l}\text { Solar } \\
\text { Radiation } \\
\text { and } \\
\text { Reflected } \\
\text { Radiation }\end{array}$ & $\begin{array}{l}\text { directly shading a person, by reducing } \\
\text { long-wave emission from courtyard } \\
\text { surfaces, and by limiting the amount } \\
\text { of solar radiation reflected from them. }\end{array}$ \\
\hline & Grass & $\begin{array}{l}\text { Covered } \\
\text { Area }\end{array}$ & $\begin{array}{l}\text { Reduces } \\
\text { Reflected } \\
\text { Radiation }\end{array}$ & $\begin{array}{l}\text { Grass surface contributes to comfort } \\
\text { due to the lower radiative surface } \\
\text { temperature and reduced emission of } \\
\text { long-wave radiation. }\end{array}$ \\
\hline [40] & Tree & $\begin{array}{l}\text { Covered } \\
\text { Area }\end{array}$ & $\begin{array}{l}\text { Reduces Air } \\
\text { Temperature }\end{array}$ & $\begin{array}{l}\text { Areas with mature trees are reaping } \\
\text { substantially more microclimate } \\
\text { benefits or urban heat island } \\
\text { reduction than areas that "just" have } \\
\text { young trees, smaller trees, and grassy } \\
\text { parks. }\end{array}$ \\
\hline \multirow[t]{2}{*}{ [41] } & Tree & $\begin{array}{l}\text { Covered } \\
\text { Area }\end{array}$ & $\begin{array}{l}\text { Reduces } \\
\text { Surface } \\
\text { Temperature }\end{array}$ & $\begin{array}{l}\text { Trees and shrubs mean maximum soil } \\
\text { temperature was } 5.7^{\circ} \mathrm{C} \text { lower than } \\
\text { lawn area. }\end{array}$ \\
\hline & Shrub & $\begin{array}{l}\text { Covered } \\
\text { Area }\end{array}$ & $\begin{array}{l}\text { Reduces } \\
\text { Surface } \\
\text { Temperature }\end{array}$ & $\begin{array}{l}\text { Trees and shrubs mean maximum soil } \\
\text { temperature was } 5.7^{\circ} \mathrm{C} \text { lower than } \\
\text { lawn area. }\end{array}$ \\
\hline [42] & Tree & $\begin{array}{l}\text { Covered } \\
\text { Area }\end{array}$ & $\begin{array}{l}\text { Reduces } \\
\text { Surface } \\
\text { Temperature }\end{array}$ & $\begin{array}{l}\text { Shade from trees reduced the } \\
\text { temperature of outer building } \\
\text { surfaces. }\end{array}$ \\
\hline [43] & Tree & $\begin{array}{l}\text { Covered } \\
\text { Area }\end{array}$ & $\begin{array}{l}\text { Reduces Air } \\
\text { Temperature }\end{array}$ & $\begin{array}{l}\text { The air temperatures were reduced by } \\
\text { up to } 2.27^{\circ} \mathrm{C} \text { relative to the current } \\
\text { condition when the number of trees } \\
\text { was increased by } 20.23 \% \text {. }\end{array}$ \\
\hline
\end{tabular}

The guidelines outlined by DID [27, 28] highlights the effectiveness of various vegetation specification so as to meet the specific objectives for a riparian buffer zone. For instance, the height of grass cover affects travel time of run-off flows, as specified by ranges: short grass at $<150 \mathrm{~mm}$ and tall grass at $\geq 150 \mathrm{~mm}$. This aids in specific implementation and maintenance on site to achieve the objective target and purposes. On the other hand, guidelines retrieved from NRE and JLN $[29,30]$ appear to emphasise more on biodiversity, vegetation species, and aesthetic values, whereas guidelines obtained by JPBD [31] seem to focus more on the overall planning associated to urban river spatial, as well as other probable functions. In addition, the findings reported in [40-41] signify that matured tree substantially enhances thermal surroundings and grass-covered areas do decrease the temperature, but the benefit is maximised if coupled with shading by trees.

Table 2 shows that all types of vegetation do have a significant role as the riparian buffer zone, apart from reducing thermal effect. In this simplified table, all urban riparian guidelines appear to place more focus on the functions of grass and shrubs in a riparian buffer zone. Meanwhile, function of tree are limited to covered area and area width. Although the functions of trees seem to be in scarcity, unlike grass and shrub, more emphasis is given for its detailed physical specification, such as tree trunk height, canopy density, and canopy width. This also illustrates that various vegetation physical aspects do benefit the river health and the local microclimate. Nevertheless, the findings portray that studies, specifically on shrubs, require further investigation in terms of thermal reduction. 
Table 2. Vegetation Type, Specification and Function

\begin{tabular}{|c|c|c|c|c|c|c|c|c|c|c|c|c|c|c|c|}
\hline \multirow[t]{3}{*}{ Function } & \multicolumn{15}{|c|}{ Vegetation Type and Specification } \\
\hline & \multicolumn{4}{|c|}{ Grass } & \multicolumn{3}{|c|}{ Shrub } & \multicolumn{8}{|c|}{ Tree } \\
\hline & 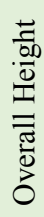 & 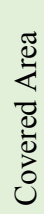 & 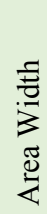 & $\frac{3}{5}$ & 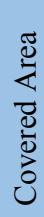 & 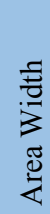 & 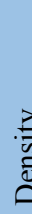 & & & & & 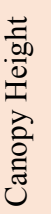 & 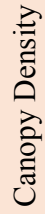 & 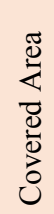 & 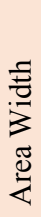 \\
\hline
\end{tabular}

\section{Function as Riparian Buffer Zone}

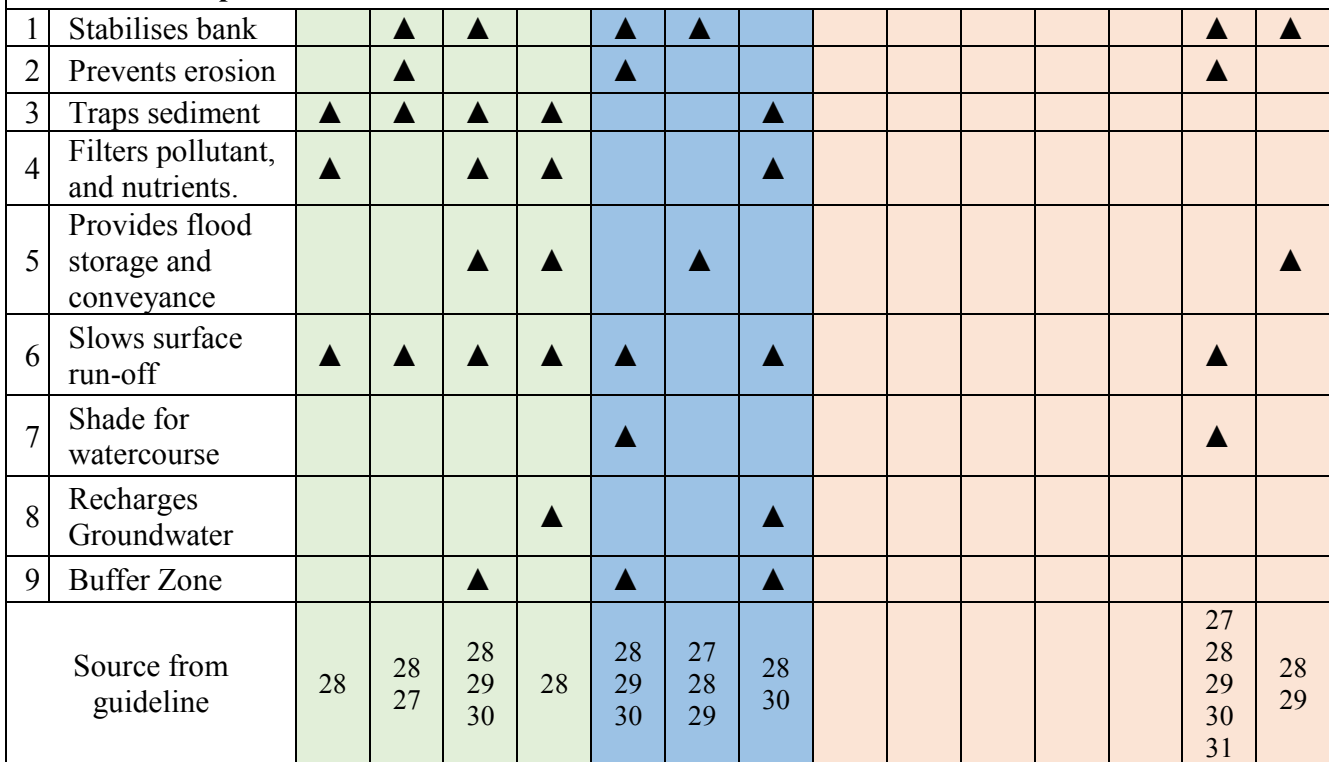

\section{Function as Thermal Reduction}

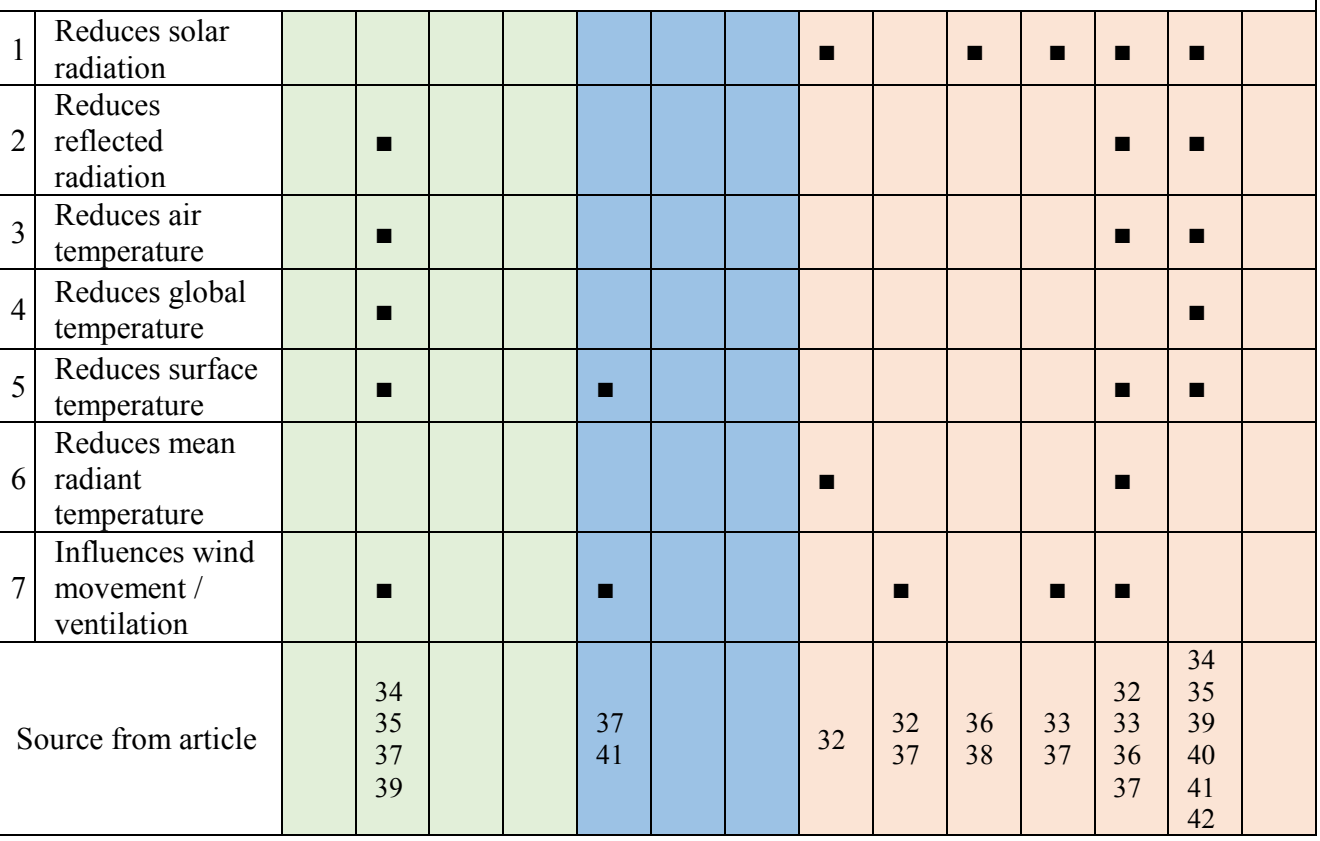




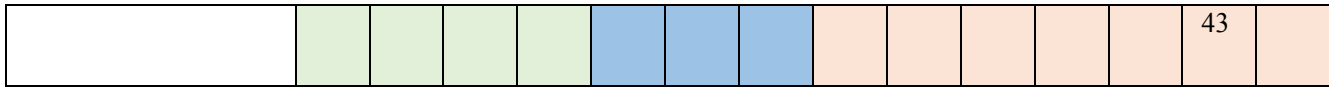

Furthermore, this table can serve as an indicator. For example, in observing the river reserve, if the area is completely covered by grass, based on Table 2, the general insight on its ability to trap sediments is highlighted. However, if the site consists of patches of bare soil area, then it might lack in terms of sediments trap functions. Such tabulation can serve as a general overview of the site condition. Hence, detailed investigation is still required to comprehend its trapping efficiency further.

This table also stipulates that by abiding with the provided guidelines, which are initially meant for river and water quality needs, it indirectly addresses issues related to urban microclimate. In the case of grass-covered areas, they do trap not only sediments, but also reduce surface, air, and global temperatures, as well as reflected radiation. Moreover, solving a problem by placing focus only on one specification is inadequate, mainly because each one is related to one another. Besides, in attaining benefits and objectives, if one benefit is achieved, indirectly another benefit will also be attained. With that, this study intends to determine the potential of urban riparian vegetation as a passive cooling strategy, in which the vegetation technical requirement for river buffer does have the ability to function as thermal reduction. This finding further contributes to local authorities and other practitioners so as to elaborate a more substantial urban river reserve maintenance and management based on its vegetation physical structure, as well as its ability in altering heat transfer mechanisms.

\section{Conclusion}

This approach can be employed to facilitate riparian area towards attaining their multiple functions and benefits, especially with a focus on vegetation physical structure. Appropriate site planning has the ability to preserve the environment through conservation and preservation techniques, aside from maximising vegetation utilisation for more integrated functions. This research also shows that for urban riparian, a combination of grasses, shrubs, and shade trees with high trunks is the most appropriate vegetation combination, particularly in hot and humid regions. It also illuminates that vegetation types and physical specification should be adhered to and further extended in future. Moreover, much attention is required regarding its implementation in terms of composition scheme and maintenance strategy. Furthermore, a more comprehensive vegetation planning based on site-specific consideration is needed, instead of a simple duplication from others, which reflect successful and improved areas. Nevertheless, other additional attributes linked to vegetation, such as root condition and root structure, should be further explored. These findings, hence, serve as a basis to integrate passive cooling strategies by embedding vegetation requirements for urban river reserve in Malaysia.

The authors would like to express their appreciation to Zamalah UTM for providing scholarship to the first author.

\section{References}

1. E. Tabacchi, L. Lambs, H. Guilloy, A. M. Planty-Tabacchi, E. Muller, and H. Décamps, Hydrol. Process. 14, 2959 (2000).

2. M. G. Dosskey, P. Vidon, N. P. Gurwick, C. J. Allan, T. P. Duval, and R. Lowrance, JAWRA J. Am. Water Resour. Assoc. 46, 261 (2010). 
3. L. Ran, X. Lu, and J. Xu, Crit. Rev. Environ. Sci. Technol. 43, 1384 (2013).

4. A. Aflaki, M. Mirnezhad, A. Ghaffarianhoseini, A. Ghaffarianhoseini, H. Omrany, Z.

H. Wang, and H. Akbari, Cities 62, 131 (2017).

5. R. Sun and L. Chen, Ecosyst. Serv. 23, 38 (2017).

6. P. H. Ibrahim, M. Md Dali, and S. Y. Muhammad Yusoff, J. Sustain. Dev. 6, 16 (2013).

7. N. S. Abdul Latip, T. Heath, S. Shamsuddin, M. S. Liew, and K. Vallyutham, in Int. Conf. Sustain. Build. Infrastruct. (ICSBI), Part World Eng. Sci. Technol. Congr. (Kuala Lumpur, Malaysia, 2010).

8. A. R. Abdullah, Trends Anal. Chem. 14, 191 (1995).

9. M. N. Hassan, M. Awang, and A. B. Jaafar, Aquat. Ecosyst. Health Manag. 9, 269 (2006).

10. I. Lee, H. Hwang, J. Lee, N. Yu, J. Yun, and H. Kim, Ecol. Modell. 353, 167 (2017).

11. M. Keshavarzifard, M. P. Zakaria, S. Keshavarzifard, and R. Sharifi, Pertanika J. Sci. Technol. 26, 95 (2018).

12. A. Binti, S. Bond, and J. McDonagh, Pacific Rim Prop. Res. J. 17, 511 (2011).

13. I. Naubi, N. H. Zardari, S. M. Shirazi, N. Farahen, B. Ibrahim, and L. Baloo, Pol. J. Environ. Stud. 25, 231 (2016).

14. C. N. Weng, Int. J. Water Resour. Dev. 28, 343 (2012).

15. E. A. Hathway and S. Sharples, Build. Environ. 58, 14 (2012).

16. V. Iakovoglou, D. Gounaridis, and G. N. Zaimes, Int. J. Innov. Sustain. Dev. 7, 271 (2013).

17. S. R. Omar and J. Sohaili, in 2018 Int. Conf. Green Energy Appl. (Singapore, 2018), pp. 45-50.

18. N. Geetha and R. Velraj, Energy Educ. Sci. Technol. Part A Energy Sci. Res. 29, 913 (2012).

19. A. Dawodu and A. Cheshmehzangi, Energy Procedia 105, 3411 (2017).

20. K. Krippendorff, Content Analysis: An Introduction to Its Methodology (2004).

21. S. Elo, M. Kääriäinen, O. Kanste, T. Pölkki, K. Utriainen, and H. Kyngäs, SAGE Open 4, 215824401452263 (2014).

22. S. Elo and H. Kyngäs, J. Adv. Nurs. 62, 107 (2008).

23. B. D. Prasad, Res. Methods Soc. Work pp. 173 (2008).

24. E. Edwards, Bothalia 14, 705 (1983).

25. Z. Abidin, N. Arbina, and G. Lee, in Proc. Int. Fed. Landsc. Archit. 47th World Congr. (Suzhou, China, 2010).

26. R. Uribe and E. Manzur, Int. J. Advert. 31, 907 (2012).

27. Department of Irrigation and Drainage Malaysia, Garis Panduan Pembangunan Melibatkan Sungai Rezab Sungai (Department of Irrigation and Drainage (DID) Malaysia, Kuala Lumpur, Malaysia, 2012).

28. Department of Irrigation and Drainage Malaysia, Urban Stormwater Management Manual for Malaysia MSMA, 2ndEdition ed. (Department of Irrigation and Drainage (DID) Malaysia, Kuala Lumpur, Malaysia, 2012).

29. Ministry of Natural Resources and Environment, Managing Biodiversity in the Riparian Zone (Ministry of Natural Resources and Environment (NRE) Malaysia, 2009).

30. Jabatan Landskap Negara, in Garis Pandu. Landsk. Negara, 2nd Ed (Jabatan Landskap Negara (JLN) Kementerian Kesejahteraan Bandar Perumahan dan Kerajaan Tempatan, 2008), pp. 36-50.

31. Jabatan Perancangan Bandar dan Desa Semenanjung Malaysia Kementerian Kesejahteraan Bandar Perumahan dan Kerajaan, Garis Panduan Perancangan Kawasan Rizab Sungai Sebagai Sebahagian Tanah Lapang Awam (Jabatan 
Perancangan Bandar dan Desa (JPBD) Semenanjung Malaysia, 2002).

32. T. E. Morakinyo, L. Kong, K. K. L. Lau, C. Yuan, and E. Ng, Build. Environ. 115, 1 (2017).

33. C. M. Hsieh, F. C. Jan, and L. Zhang, Urban For. Urban Green. 18, 126 (2016).

34. D. Armson, P. Stringer, and A. R. Ennos, Urban For. Urban Green. 11, 245 (2012).

35. A. Chatzidimitriou and S. Yannas, Energy Build. 108, 156 (2015).

36. M. F. Shahidan, M. K. M. Shariff, P. Jones, E. Salleh, and A. M. Abdullah, Landsc. Urban Plan. 97, 168 (2010).

37. B. Givoni, Atmos. Environ. Part B, Urban Atmos. 25, 289 (1991).

38. L. Shashua-Bar and M. E. Hoffman, Energy Build. 31, 221 (2000).

39. L. Shashua-Bar, D. Pearlmutter, and E. Erell, Int. J. Climatol. 31, 1498 (2011).

40. A. Y. Davis, J. Jung, B. C. Pijanowski, and E. S. Minor, Appl. Geogr. 71, 106 (2016).

41. J. L. Edmondson, I. Stott, Z. G. Davies, K. J. Gaston, and J. R. Leake, Sci. Rep. 6, 33708 (2016).

42. H. Akbari, D. M. Kurn, S. E. Bretz, and J. W. Hanford, Energy Build. 25, 139 (1997).

43. M. Srivanit and K. Hokao, Build. Environ. 66, 158 (2013). 